\title{
Aryl hydrocarbon receptor, a tumor grade-associated marker of oral cancer, is directly downregulated by polydatin: A pilot study
}

\author{
MANUELA MARTANO $^{1 *}$, PAOLA STIUSO ${ }^{2 *}$, ANGELO FACCHIANO ${ }^{3}$, SALVATORE DE MARIA $^{4,5}$, \\ DANIELA VANACORE ${ }^{2}$, BRUNELLA RESTUCCI ${ }^{1}$, CORRADO RUBINI $^{6}$, MICHELE CARAGLIA ${ }^{2}$, \\ GIAMPIETRO RAVAGNAN ${ }^{5}$ and LORENZO LO MUZIO ${ }^{7}$
}

\author{
${ }^{1}$ Department of Veterinary Medicine and Animal Production, University Federico II of Naples, I-80137 Naples; \\ ${ }^{2}$ Department of Biochemical, Biophysical and General Pathology, Università della Campania 'Luigi Vanvitelli', I-80138 Naples; \\ ${ }^{3}$ CNR-ISA National Research Council, Institute of Food Science, I-83100 Avellino; ${ }^{4}$ Ministry of Education, \\ University and Research (MIUR); CPIA Naples City 1, 'Pasquale Scura' Polo Tecnico Fermi Gadda, \\ I-80141 Naples; ${ }^{5}$ Glures Spin-off CNR-IFT National Research Council, Translational Institute of Pharmacology, \\ I-00133 Rome; ${ }^{6}$ Department of Clinical Sciences, Polytechnic University of Marche, I-60121 Ancona; \\ ${ }^{7}$ Department of Clinical and Experimental Medicine, University of Foggia, I-71122 Foggia, Italy
}

Received July 3, 2017; Accepted January 12, 2018

DOI: $10.3892 / o r .2018 .6555$

\begin{abstract}
Oral squamous cell carcinoma (OSCC) is one of the most aggressive and deadliest tumors worldwide. The aryl hydrocarbon receptor (AHR) is a nuclear transcription factor known as a dioxin receptor and mediates the toxic effects of industrial contaminants. In addition, AHR has been implicated in multiple cellular processes and its expression has been shown to play a critical role in tumorigenesis, including human oral cancer cell lines. In the present study, we evaluated the expression of AHR/HSP-90 in 25 formalin-fixed, paraffinembedded human oral cancer specimens by IHC analysis. CYP1A1 expression was regarded as an AHR reporter gene. The data indicated a complete correlation between AHR expression and cancer grade enabling us to propose AHR as a prognostic marker of oral cancer. Moreover, in OSCC cell line CAL27, we observed the modulatory effect of polydatin, a widespread natural substance and direct precursor of resveratrol, on AHR expression. A computational approach was performed to predict the site of interaction of polydatin on the AHR surface. Our studies confirm the involvement of AHR signaling in the clinicopathological specimens of oral cancer and suggest the use of polydatin for oral cancer prevention.
\end{abstract}

Correspondence to: Dr Salvatore De Maria, Ministry of Education, University and Research (MIUR); CPIA Naples City 1, 'Pasquale Scura' Polo Tecnico Fermi Gadda, Corso Malta 141, I-80141 Naples, Italy

E-mail: salvatore.demaria4@istruzione.it

*Contributed equally

Key words: IHC, AHR, HSP-90, resveratrol, polydatin

\section{Introduction}

The aryl hydrocarbon receptor (AHR) is a nuclear transcription factor known as a dioxin receptor and mediates the toxic effects of industrial contaminants such as polycyclic aromatic hydrocarbons, the forefather being 2,3,7,8-tetrachlorodibenzo- $p$-dioxin or TCDD. The effect of TCDD upon AHR activation on cancer stages are well known $(1,2)$. Prior to ligand binding AHR is found in the cytosol in the form of a multiprotein complex with a sedimentation coefficient of 9 Svedberg (AHRC-9S). The complex consists of five different polypeptides and six proteins including AHR, heat shock protein 90-homodimer (two HSP-90 proteins), P23 (a prostaglandin E synthase with co-chaperonin activity vs. HSP-90), SRC kinase (a tyrosin kinase homologous to viral oncogenes of the Rous sarcoma) and AHR interacting protein (AIP) (3). Upon activation by a wide range of environmental toxins, AHR translocates to the nucleus and heterodimerizes with the AHR nuclear translocator protein (ARNT). Finally, the AHR-ARNT complex binds DNA to the xenobiotics responsive element (XRE) of the promoters, resulting in the transcriptional activation of genes, such as cytochrome P450 (CYP450). The human CYP superfamily (CYP1, CYP2 and CYP3) contains 57 functional genes, and play an important role in the metabolism of therapeutic drugs, other xenobiotics, and various endogenous compounds. Several of these CYPs (e.g., CYP1A1 and 1A2) are involved in the metabolism and bioactivation of carcinogens. Cytochrome P450 1A1, a well-known aryl hydrocarbon hydroxylase, is one of the most important enzymes responsible for the metabolism of environmental pollutants and many aromatic compounds involved in tumorigenesis (4).

Since AhR activation and subsequent induction of AhR-regulated P450 enzymes (CYP1A1, CYP1B1) have been implicated in oral squamous cell carcinoma (OSCC) (5), we investigated the role of AHR and HSP-90 in OSCC 
tumorigenesis and progression. The expression of AHR by immunohistochemical staining of OSCC formalin-fixed, paraffin-embedded (FFPE) tissues was determined and its correlation with different tumor grades was assessed. As control of the transcriptional activation of AHR downstream stimulated genes we also investigated CYP1A1 (6).

Polydatin (trans-piceid 3,5,4'-trihydroxystilbene-3-O- $\beta$-Dglucopyranoside) is a widespread natural substance contained in red wine and a direct precursor of resveratrol, a well-known AHR inhibitor $(7,8)$. The glucopyranoside ring of polydatin influences the pharmocodynamics (9) and pharmacokinetics $(10,11)$ resulting in better biodisponibility in respect to resveratrol.

Polydatin was also demonstrated to act as an antitumoral substance by stimulation of apoptosis in leukemia, lung cancer, colorectal cancer cell lines, and ovarian cancer (12-17).

In the present study, we confirmed the effect of polydatin in the CAL27 cell line, a model of oral carcinoma to establish the effects on AHR expression and monitoring the AHR transcriptional factor activity using CYP1A1 as an AHR reporter gene. Moreover, we monitored the effect of polydatin on HSP-90, a component of the AHR complex, essential for Ah receptor signaling $(18,19)$.

In a previous study by computational analysis, the binding site for ligands on the human AHR surface was proposed (20). With a similar computational approach, we present our results relative to docking studies on the direct interaction between polydatin and human AHR.

\section{Materials and methods}

Patients and tissue samples. Twenty-five OSCC patients were enrolled in the present study. Tumor tissues were collected at the time of resection, and informed written consent for the use of sample tissues was obtained from all patients. We did not seek ethics committee approval, as all data analyzed were collected as part of routine diagnosis and treatment, and all patients were diagnosed and treated according to institutional guidelines and agreements.

Moreover, the present study does not report on the use of experimental therapies. The baseline characteristics of the OSCC patients investigated in the study are reported in Table I. Twenty-five samples of hOSCC from various sites of oral regions were collected. Each tissue sample was fixed in $10 \%$ neutral-buffered formalin, embedded in paraffin, routinely processed and stained in hematoxylin and eosin. Each sample was independently graded by two observers: grade $1(11 / 25)$, grade $2(8 / 25)$ and grade $3(6 / 25)$ OSCC, according to the Histological Grading System proposed by Bryne et al (21).

Immunoperoxidase staining. Sections were de-paraffinized in xylene, dehydrated in graded alcohols and washed in $0.01 \mathrm{M}$ phosphate-buffered saline (PBS). Endogenous peroxidase was blocked with hydrogen peroxide $0.3 \%$ in absolute methanol for $30 \mathrm{~min}$. The streptavidin-biotin-peroxidase method (LSAB kit; Dako, Glostrup, Denmark) was used. Antigen enhancement was performed by pretreatment with microwave heating in citrate buffer, $\mathrm{pH} 6.0$, twice for $5 \mathrm{~min}$ at $750 \mathrm{~W}$. As primary antibodies, polyclonal rabbit anti-human AHR receptor (clone H-211) (Santa Cruz Biotechnology, Inc., Dallas, TX, USA),
Table I. Baseline characteristics of the OSCC patients investigated in the study.

\begin{tabular}{lrr}
\hline & \multicolumn{2}{c}{ Patients } \\
\cline { 2 - 3 } Characteristics & No. & \\
\hline Age, years & & \\
$\leq 60$ & 19 & 24 \\
$>60$ & & \\
Sex & 15 & 60 \\
Male & 10 & 40 \\
Female & & 36 \\
N-regional lymph node status & 9 & 64 \\
Negative & 16 & \\
Positive & & 34 \\
Histological grade & 11 & 24 \\
Grade 1 & 8 & 72 \\
Grade 2 & 6 & 28 \\
Grade 3 & & \\
Smoking history & & \\
Yes & 18 & \\
No & 7 & \\
\hline
\end{tabular}

OSCC, oral squamous cell carcinoma.

polyclonal rabbit anti-human cytochrome $\mathrm{P} 450$ (clone A1A) and HSP-90 (AB 13492) (both from Abcam, Cambridge, UK) were used; they were diluted 1:100 and 1:200 in PBS and applied overnight at $4^{\circ} \mathrm{C}$. The immunolabeling procedure included negative control sections incubated with normal serum, instead of the primary antibody. The MACH1 Universal HPR-Polymer Detection kit (Biocare Medical LLC, Concord, CA, USA) was used as the secondary antibody. In order to visualize immunolabeling, 3'3'-diaminobenzidine tetrahydrochloride was used as a chromogen, with hematoxylin as a counterstain.

Scoring of immunoreactivity and statistical analysis. The intensity of immunolabeling in each specimen, for each antibody, was scored by two independent observers, under blinded conditions, as performed in a previous study (22). For each tumor, 20 fields were examined at a magnification of x200 (20X objective, 10X ocular), and the immunosignal was scored from absent to strong, as follows: n.a., not assessed; -, negative staining; +/-, weak immunolabeling; +, moderate immunolabeling; ++ , strong immunolabeling.

Moreover, the mean percentage of positive cells in 10 high-power microscopic fields was determined using a scale of 1-4 as follows: 1 indicated the staining of $0-25 \%$ of cells, and 2, 3 and 4 indicated 25-50, 50-75 and 75-100\% staining, respectively.

Cell culture. Human tongue squamous cell carcinoma cell line CAL27 was obtained from the American Tissue Culture Collection (ATCC; Rockville, MD, USA). The cells were grown 
in DMEM with $10 \%$ fetal bovine serum (FBS) (both from Gibco; Thermo Fisher Scientific, Inc., Waltham, MA, USA), $100 \mathrm{U} / \mathrm{ml}$ penicillin and $100 \mu \mathrm{g} / \mathrm{ml}$ streptomycin in a humidified $37^{\circ} \mathrm{C}$ incubator with $5 \% \mathrm{CO}_{2}$. After incubation for $4 \mathrm{~h}$ in Dulbecco's modified Eagle's medium (DMEM) with 10\% FBS, the cells were washed with $1 \%$ phosphate-buffered saline (PBS) to remove unattached dead cells, and were incubated for $24 \mathrm{~h}$ with different concentrations (range $0-150 \mu \mathrm{M}$ ) of polydatin (trans-5,4'-dihydroxystilbene-3-O- $\beta$-D-glucopy-ranoside).

Cell viability. We assessed the sensitivity of the cell lines tested to polydatin using a microplate colorimetric assay that measures the ability of viable cells to transform a soluble tetrazolium salt (MTT) to an insoluble purple formazan precipitate. Cells were plated at the appropriate density $\left(5 \times 10^{3} \mathrm{Cal}-27\right.$ cells) in 96-well microtitre plates. After $4 \mathrm{~h}$, cells were exposed to definite concentrations of polydatin for $24 \mathrm{~h}$. Then, $50 \mu \mathrm{l}$ of MTT $(1 \mathrm{mg} / \mathrm{ml})$ and $200 \mu \mathrm{l}$ of medium were added to the cells in each well. After a $4 \mathrm{~h}$ incubation at $37^{\circ} \mathrm{C}$, the medium was removed, and the formazan crystals were solubilized by adding $150 \mu \mathrm{l}$ of DMSO and by mixing it in an orbital shaker for $5 \mathrm{~min}$. The absorbance at $570 \mathrm{~nm}$ was measured by Bio-Rad 550 microplate reader (Bio-Rad Laboratories, Milan, Italy). Experiments were performed in triplicate. As a control, $0.5 \%$ DMSO was added to untreated cells.

Western blot analysis. Cal-27 cells were grown for $24 \mathrm{~h}$ with or without polydatin at the concentrations reported in Fig. 5 legend. The cells were washed twice with ice-cold PBS/BSA, scraped and centrifuged for $30 \mathrm{~min}$ at $4^{\circ} \mathrm{C}$ in $1 \mathrm{ml}$ of lysis buffer ( $1 \%$ Triton, $0.5 \%$ sodium deoxycholate, $0.1 \mathrm{M} \mathrm{NaCl}$, $1 \mathrm{mM}$ EDTA, pH 7.5, $10 \mathrm{mM} \mathrm{Na}_{2} \mathrm{HPO}_{4}, \mathrm{pH} 7.4,10 \mathrm{mM}$ PMSF, $25 \mathrm{mM}$ benzamidin, $1 \mathrm{mM}$ leupeptin, $0.025 \mathrm{U} / \mathrm{ml}$ aprotinin). Equal amounts of cell proteins were separated by SDS-PAGE. The proteins on the gels were electrotransferred to nitrocellulose and reacted with polyclonal rabbit anti-human AHR receptor (clone H-211) (Santa Cruz Biotechnology), polyclonal rabbit anti-human cytochrome P450 (clone A1A) and HSP-90 (AB 13492) (both from Abcam) as recommended by the manufacturer. This step was followed by incubation with corresponding horseradish peroxidase (HRP)-conjugated secondary antibody. Protein bands were detected by chemiluminescence detection reagents (Millipore Corporation, Billerica, MA, USA). The protein bands were quantified using the NIH ImageJ system (National Institute of Health, Bethesda, MD, USA). All the western blot analyses were repeated three times. Western blotting for tubulin was used as an internal control. To quantify the results, the relative amount of each protein was determined.

Molecular simulations. Molecular docking approach was used to simulate interaction of polydatin and the human AHR ligand binding domain. The structure of polydatin was obtained from PubChem database (https://pubchem.ncbi.nlm.nih.gov/). The structure of human AHR ligand binding domain used in the simulations was that described in a previous study (20). In brief, the amino acid sequence of human Ah receptor obtained from Uniprot (Accession no. P35869) was restricted to the region between residue 290 and 390 . Secondary structures were calculated with the DSSP program and three-dimen- sional models of human AHR-polydatin were elaborated using MODELLER 9v7 version and evaluated for stereo-chemical with PRO-CHECK program. The energy profiles were elaborated applying PROSAII program. Docking procedure was performed using AutoDock 4.2 and ADTools for preparation of molecules and parameters files, according to the procedures used in a previous study (20). At least 100 docking determination using Lamarckian genetic algorithm with the default parameters were performed, with a maximum of $2.5 \times 10^{6}$ energy evaluations. Only the conformational clusters most populated and with lower binding energy $(E b)$ were utilized in docking analysis and clustered using an root-mean-square distance (RMSD) value of $2.0 \AA$ A . Molecular images were generated by BIOVIA Discovery Studio (Dassault Systèmes).

\section{Results}

Immunohistochemistry. We examined the intensity of immunolabeling, the percentage of positive cells and the localization of AHR, CYP1A1 and HSP-90 in 25 OSCCs, and we report the results in Table II.

AHR expression. In all neoplastic samples, AHR expression was presented by a cytoplasmic pattern; however, $50 \%$ of grade $2(4 / 8)$ and $80 \%$ of grade $3(4 / 6)$ OSCCs showed nuclear immunostaining Moreover, the intensity of immunostaining and the percentage of positive epithelial neoplastic cells were increased according to the differentiation grade of the tumor. Particularly, in almost all G1 tumors (10/11), the percentage of positive epithelial neoplastic cells ranged from 25 to $50 \%$ and the intensity of immunostaining was weak (Fig. 1). The majority of grade 2 tumors (7/8) showed a percentage of positive epithelial neoplastic cells ranging from 50 to $75 \%$ and a moderate intensity of immunostaining (Fig. 2). Grade 3 tumors showed a moderate $(2 / 6)$ to strong immunoreactivity (4/6) and $80-100 \%$ of epithelial neoplastic cells were positive in almost all samples (5/6) (Fig. 3).

CYPIA1 and HSP-90 expression. In all neoplastic samples, CYP1A1 and HSP-90 expression showed a cytoplasmic pattern, particularly in grade 1 and 2, while $50 \%$ of grade 3 OSCCs (3/6) showed a nuclear localization of HSP-90. The intensity of immunostaining and the percentage of epithelial positive neoplastic cells were increased proceeding from grade 1 OSCC, in which only $30 \%$ of cells were weakly positive for both CYP1A1 (Fig. 2A) and HSP-90 (Fig. 3A), to grade 2 (Figs. 2B and 3B) in which almost all samples (6/8) showed a moderate immunolabeling in $50-75 \%$ neoplastic cells, proceeding to grade 3 OSCC, in which $75-100 \%$ of neoplastic cells were strongly positive in 5/6 samples (Figs. 2C and 3C).

In vitro effect of polydatin on AHR, CYPlAl and HSP-90 expression in human tongue squamous cell carcinoma CAL27 cells. The effects of trans-polydatin on the proliferation of human tongue squamous cell carcinoma CAL27 cells was evaluated by MTT assay (see section reported in 'Materials and Methods'). Polydatin induced a dose-dependent growth inhibition after $24 \mathrm{~h}$ and the $\mathrm{IC}_{50}$ (inhibition of $50 \%$ of cell growth) was reached at the $20 \mu \mathrm{M}$ concentration (Fig. 4). 
Table II. IHC of Ah receptor, cytochrome P450 and HSP-90 in 25 OSCC tissue samples.

\begin{tabular}{|c|c|c|c|c|c|c|c|}
\hline \multirow[b]{2}{*}{ Sample $\mathrm{a}^{\mathrm{a}}$} & \multirow[b]{2}{*}{ Grade } & \multicolumn{2}{|r|}{ AhR } & \multicolumn{2}{|r|}{ CYP1A1 } & \multicolumn{2}{|c|}{ HSP-90 } \\
\hline & & $\begin{array}{c}\text { Intensity } \\
\mathrm{IHC}^{\mathrm{b}}\end{array}$ & $\%$ Positive cells ${ }^{\mathrm{c}}$ & $\begin{array}{c}\text { Intensity } \\
\text { IHC }^{\mathrm{b}}\end{array}$ & $\%$ Positive cells ${ }^{\mathrm{b}}$ & $\begin{array}{c}\text { Intensity } \\
\mathrm{IHC}^{\mathrm{b}}\end{array}$ & $\%$ Positive cells \\
\hline $\mathrm{T} 1$ & 1 & $+/-$ & 2 & $+/-$ & 2 & $+/-$ & 2 \\
\hline $\mathrm{T} 2$ & 1 & $+/-$ & 2 & + & 2 & ++ & 2 \\
\hline $\mathrm{T} 3$ & 1 & $+/-$ & 2 & + & 2 & $+/-$ & 2 \\
\hline $\mathrm{T} 4$ & 1 & $+/-$ & 2 & + & 2 & + & 2 \\
\hline $\mathrm{T} 5$ & 1 & $+/-$ & 2 & $+/-$ & 2 & $+/-$ & 2 \\
\hline T6 & 1 & + & 2 & ++ & 2 & $+/-$ & 2 \\
\hline $\mathrm{T} 7$ & 1 & $+/-$ & 2 & $+/-$ & 2 & + & 2 \\
\hline $\mathrm{T} 8$ & 1 & $+/-$ & 1 & $+/-$ & 2 & + & 2 \\
\hline T9 & 1 & $+/-$ & 2 & + & 2 & $+/-$ & 2 \\
\hline $\mathrm{T} 10$ & 1 & $+/-$ & 2 & + & 2 & + & 2 \\
\hline T11 & 1 & $+/-$ & 2 & $+/-$ & 2 & + & 2 \\
\hline $\mathrm{T} 12$ & 2 & + & 3 & + & 2 & + & 2 \\
\hline $\mathrm{T} 13$ & 2 & + & 3 & ++ & 3 & + & 3 \\
\hline $\mathrm{T} 14$ & 2 & ++ & 3 & ++ & 3 & + & 3 \\
\hline $\mathrm{T} 15$ & 2 & + & 2 & + & 2 & + & 2 \\
\hline $\mathrm{T} 16$ & 2 & + & 3 & ++ & 3 & + & 3 \\
\hline T17 & 2 & + & 3 & ++ & 3 & + & 3 \\
\hline $\mathrm{T} 18$ & 2 & + & 3 & + & 3 & + & 3 \\
\hline T19 & 2 & + & 3 & + & 3 & + & 3 \\
\hline $\mathrm{T} 20$ & 3 & ++ & 4 & + & 4 & ++ & 4 \\
\hline $\mathrm{T} 21$ & 3 & ++ & 3 & ++ & 4 & ++ & 3 \\
\hline $\mathrm{T} 22$ & 3 & + & 4 & ++ & 4 & ++ & 4 \\
\hline $\mathrm{T} 23$ & 3 & ++ & 4 & + & 3 & ++ & 4 \\
\hline $\mathrm{T} 24$ & 3 & + & 4 & ++ & 4 & ++ & 4 \\
\hline $\mathrm{T} 25$ & 3 & ++ & 4 & ++ & 4 & ++ & 4 \\
\hline
\end{tabular}

${ }^{\mathrm{a}} \mathrm{T}$, tumor sample; bintensity of immunlabeling: -, negative staining; +/-, weak immunolabeling; + , moderate immunolabeling; ++, strong immunolabeling; c\% of positive neoplastic cells; $1,0-25 \% ; 2,>25-50 \% ; 3,>50-75 \% ; 4,>75-100 \%$. IHC, immunohistochemistry.
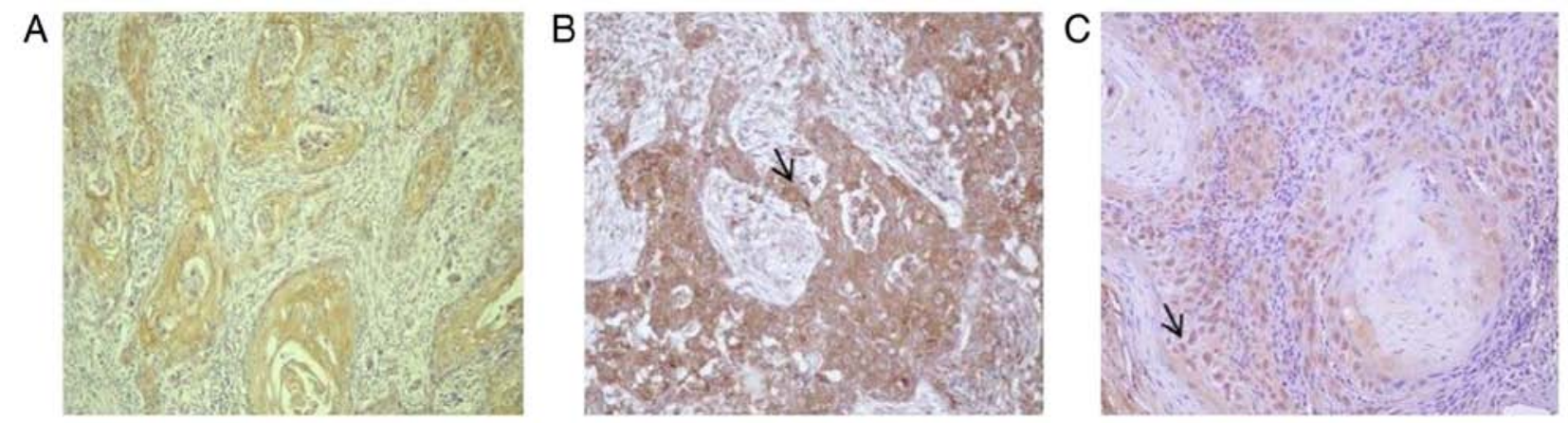

Figure 1. Immunohystochemical expression of AhR in OSCC. (A) Well-differentiated (grade 1). OSCC tissue demonstrating weak expression of the AhR protein in cytoplasm of neoplastic cells. Streptavidin-biotin peroxidase; magnification, x20. (B) Moderately differentiated (grade 2) OSCC tissue demonstrating moderate expression of the AhR protein in numerous neoplastic cells. The AhR expression was localized in almost all of the cytoplasm (arrow). Streptavidin-biotin peroxidase; magnification, x40. (C) Poorly differentiated (grade 3) OSCC tissue demonstrating strong expression of the AhR protein in almost all neoplastic cells, with a prevalent nuclear localization (arrow). Streptavidin-biotin peroxidase; magnification, x20. OSCC, oral squamous cell carcinoma.

The expression and densitometric quantification of AHR and related proteins CYP1A1 and HSP-90 in the CA127 cells before and after treatment with different concentrations of polydatin are shown (Fig. 5). The data showed that following 

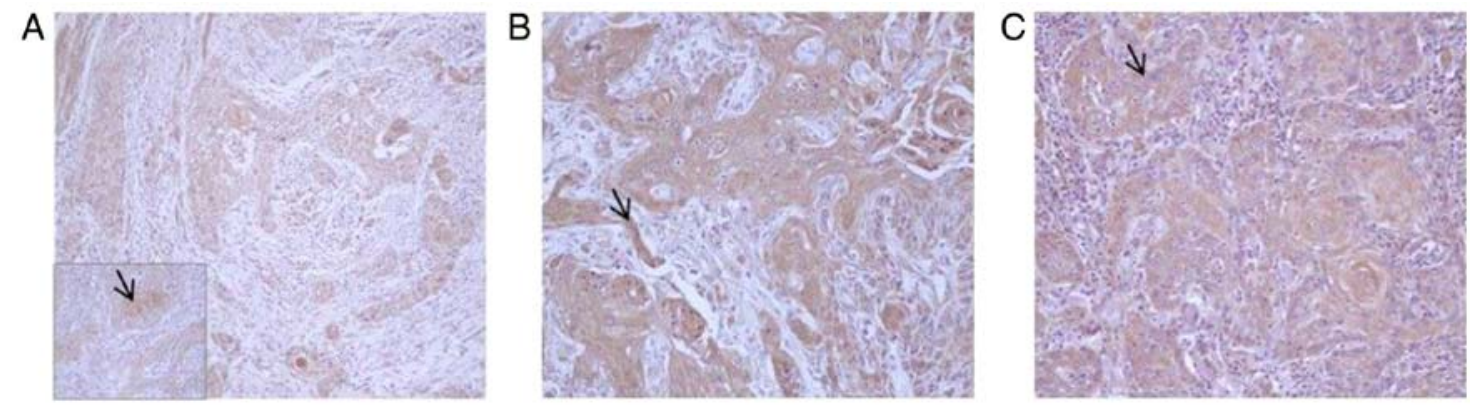

Figure 2.Immunohystochemical expression of CYP1A1 in OSCC. (A) Well-differentiated (grade 1) OSCC tissue demonstrating weak expression of the CYP1A1 protein in cytoplasm of neoplastic cells (arrow). Streptavidin-biotin peroxidase; magnification, x10; insert magnification x40. (B) Moderately differentiated (grade 2) OSCC tissue demonstrating moderate cytoplasmic expression of the CYP1A1 protein (arrow) in numerous neoplastic cells. Streptavidin-biotin peroxidase; magnification, x20. (C) Poorly differentiated (grade 3) OSCC tissue demonstrating strong expression of the CYP1A1 protein in almost all neoplastic cells, with cytoplasmic localization (arrow). Streptavidin-biotin peroxidase; magnification, x20.

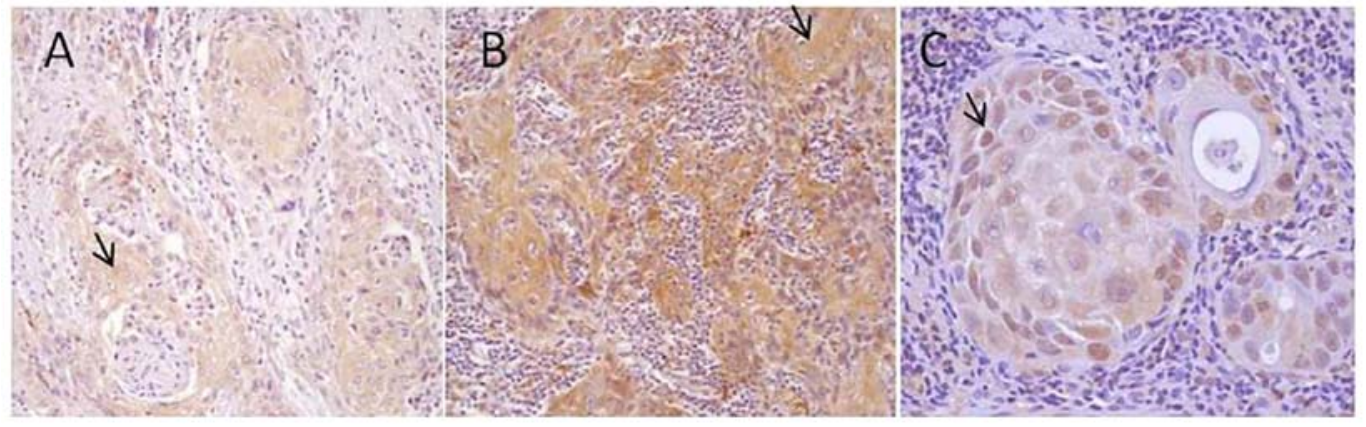

Figure 3. Immunohystochemical expression of HSP-90 in OSCC. (A) Well-differentiated (grade 1) OSCC tissue demonstrating weak expression of the HSP-90 protein in cytoplasm of few neoplastic cells (arrow). Streptavidin-biotin peroxidase; magnification, x20. (B) Moderately differentiated (grade 2) OSCC tissue demonstrating moderate to strong expression of the HSP-90 protein in numerous neoplastic cells. The HSP-90 expression was localized in almost all the cytoplasm (arrow). Streptavidin-biotin peroxidase; magnification, x20. (C) Poorly differentiated (grade 3) OSCC tissue demonstrating strong expression of the HSP-90 protein in almost all neoplastic cells, with a prevalent nuclear localization (arrow). Streptavidin-biotin peroxidase; magnification, $\mathrm{x} 40$.

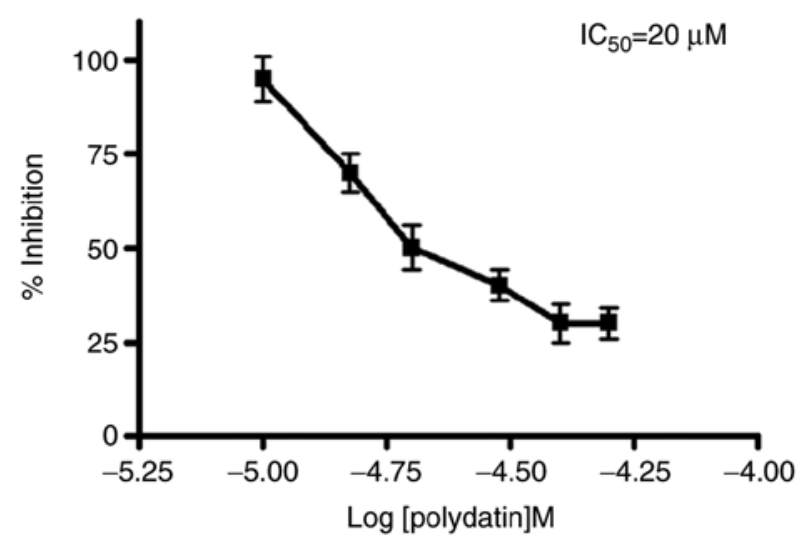

Figure 4. Effect of polydatin on the growth of human tongue squamous cell carcinoma CAL27 cells. Cell growth was evaluated by the MTT assay as described in Materials and methods. The CAL27 cells were treated for $24 \mathrm{~h}$ with increasing concentrations of polydatin at $10,15,20$ and $30 \mu \mathrm{M}$. The effects on cell growth are expressed as \% inhibition respect to the untreated cells (control).

polydatin treatment for $24 \mathrm{~h}$, a significant decrease in AHR, Cyp and HSP-90 expression in CAL27 cells was noted compared to that in the control (cells without treatment).

Molecular simulations. We simulated the interaction of polydatin with human AHR ligand binding domain, similarly

\section{A $\quad \operatorname{Ctr} 10 \mu \mathrm{M} \quad 15 \mu \mathrm{M} \quad 20 \mu \mathrm{M} \quad 30 \mu \mathrm{M}$}

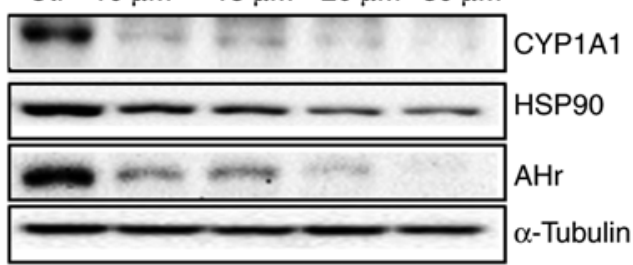

B

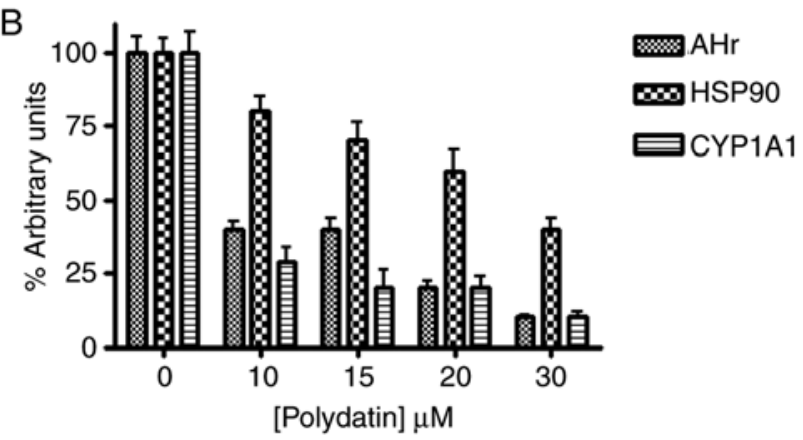

Figure 5. Effects of polydatin on AHR, HSP-90, CYP1A1 and tubulin expression. (A) The protein expression was evaluated by western blot analysis. The cells were treated with polydatin at $\mathrm{IC}_{50}$ concentrations for $24 \mathrm{~h}$, and then were processed for western blot analysis as described in 'Materials and methods'. The expression of the housekeeping protein tubulin was used as a loading control. (B) The expression levels of proteins expressed as a percentage with respect to the level of the protein in the untreated cells, used as control. Bars show the mean values \pm SD. Each experimental value reported is the mean of three different determinations. 

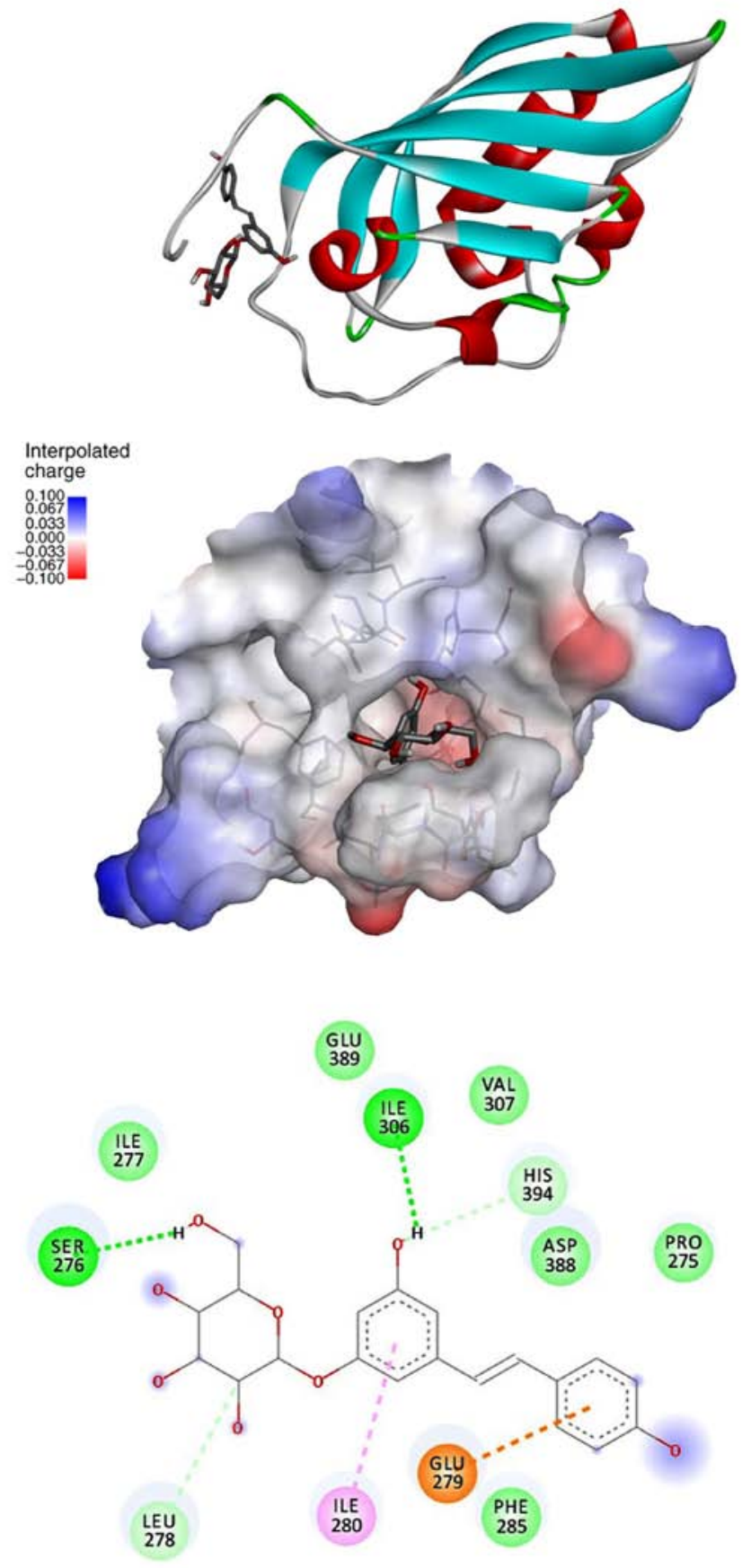

$\underset{282}{\text { THR }}$

Figure 6. Molecular simulations of the AHR and polydatin interaction. The top panel shows the ligand binding domain structure of human AHR and poyldatin in the binding site evidenced by molecular simulations. Polydatin is shown with stick representation, while human AHR is shown with backbone ribbon with $\beta$ strands in cyan, $\alpha$ helices in red, and turns in green. The middle panel shows the detail of polydatin docked into the binding site, with protein surface colored by a charge spectrum (blue for positive, red for negative charge). The bottom panel show a 2D schematization of the interaction, with reference to the amino acids involved into the binding.

to a previous study (20) in order to investigate if and how the molecules may interact. In Fig. 6 we report images to describe the results. The structure of the ligand binding domain consists of a globular domain having the typical PAS domain architecture, which consists of an anti-parallel five-strand $\beta$ sheet and an $\alpha$-helix, with a few other short secondary structure elements. Polydatin finds a suitable pocket for binding, similarly to a previous study with other ligands that are evidenced by four different possible sites (20). The preferred site is that indicated as B site in that article, and polydatin shows a mean energy of interaction of -6.51 , similar to that reported in the previous study for TCDD (dioxin), i.e, -6.2, quercetin, i.e., -6.5, and pentachlorobyphenyl, i.e., 6.54 .

\section{Discussion}

In the present study we found that AHR expression was extremely high in human oral squamous cancer (hOSC) tissues (Table I), suggesting that IHC evaluation of AHR may be considered as a prognostic marker. The influence of AHR in tumor development of hOSC is supported by a published study investigating AHR expression in cells derived from head and neck cancers and demonstrating the ability of AHR inhibitors to induce transcriptional repression of the pro-inflammatory cytokine Il-6, to counteract the drug resistance induced by ABCG2 and to repress cell migration and invasion (23). More recently, the same authors identifed three growth factors as a target of AHR, amphiregulin (AREG), epiregulin (EREG) and platelet-derived growth factor (PDGF) associated with metastatic phenotype and displaying a common DRE-like promoter motif (24). These findings are consistent with the increased AHR expression observed for other types of cancer such as gastric and non-small cell lung cancer, and mammary gland tumors (25-27). Increased AHR expression is likely to increase basal AHR activity within tumors, particularly given the systemic omnipresence of AHR agonist ligands derived from xenobiotic, dietary and microbial sources. Moreover, a correlation was found between increasing nuclear AHR protein expression and increasing tumor grade, suggesting at least for these tumors that nuclear AHR levels are predictive of a higher tumor grade.

AHR was predominantly expressed in the cytoplasm in all grade 1 and $50 \%$ of grade 2 OSCC tissues samples; on the contrary in $50 \%$ of grade 2 OSCC and all grade 3 OSCC the AHR expression was prevalently nuclear. Since AHR acts as a transcriptional regulator with nuclear translocation, the presence of nuclear AHR in grade 2 and grade 3 samples suggest a strong transcriptional activity of AHR in these high-grade tumors. Research of malignant tumors showed increased levels of AHR and its constitutive localization in the nucleus, suggesting that the AHR is chronically activated in tumors, facilitating tumor progression (28).

A large body of evidence has demonstrated that AHR is the major regulatory element for CYP1A1 expression as confirmed by our results due to high expression of AHR and CYP1A1 in high grade OSCC $(5,29,30)$. In addition, we showed that HSP-90, a related cytosolic AHR-complex protein, increased its expression from grade 1 proceeding to higher grade OSCC, and that it had prevalently a cytoplasmic localization in grade 1, grade 2 and $50 \%$ of grade 3 OSCC. In the other $50 \%$ of grade 3 OSCC, HSP-90 showed a nuclear localization, confirming what has already been demonstrated by other studies, that AHR translocates to the nucleus while in complex with HSP-90 (19). 
We studied the antitumoral effect of polydatin in vitro, on the human tongue squamous cell carcinoma cell line CAL27. Cytotoxic study of polydatin was evaluated by MTT assay and showed a $\mathrm{IC}_{50}$ value of $20 \mu \mathrm{M}$. Our western results clearly indicated a comparable inhibition of AHR, CYP1A1 and HSP-90 at all concentrations utilized ranging between 10 and $30 \mu \mathrm{M}$. Yet, while the AHR and CYP1A1 expression tended to disappear, the HSP-90 expression did not fully shutdown. This experimental evidence is ascribed to the fact that the molar ratio AHR/HSP-90 is $1 / 2$ since HSP-90 is present as a homodimer, in double copy, in the AHR-complex and HSP-90 is not exclusively part of the AHR-complex. Thus, polydatin did not act as an HSP-90 inhibitor and its decrease was AHR-dependent.

Concerning the mechanism of action of polydatin on AHR inhibition, molecular simulations suggest that this molecule may interact directly with human AHR ligand binding domain (middle panel, Fig. 6), at the same site and with similar mean energy of binding reported for dioxin, pentachlorobyphenyl, and quercetin (20). These findings suggest a direct interaction of polydatin in the active site of the human AHR where the glycosidic ring does not compromise the bond of the resveratrolic counterpart of polydatin, but that it is stabilized by Ser 276 and Leu 278 (bottom panel, Fig. 6). Polydatin is not the only glycoside derivative that enhances its inhibitory action on the AHR receptor, quercetin-6-C- $\beta$-D-glucopyranoside, a natural glycosidic analog of quercitin, was shown to interact with the active site of the human aryl receptor (31). Moreover, in this study the authors, utilizing the PC-3 cell line as a model of prostate cancer, pointed out the involvement of the Akt/mTOR pathway according to our published experiment on $\mathrm{Caco} 2$ gastrointestinal cancer cells showing the inhibitory effect of polydatin on AKT expression (16). The involvment of the AKT/mTOR pathway also leads to a autophagic input (32) which may explain the lack of expression of AHR that we registered in western analysis and that was maximum at $30 \mu \mathrm{M}$ polydatin.

In conclusion, oral squamous cell carcinoma is increasingly associated with exposure to numerous environmental carcinogens, including mostly tobacco smoke and its carcinogenic components, such as polycyclic aromatic hydrocarbons (PAHs).

These carcinogens are transformed in mutagenic compounds by catabolic activity of AHR-regulated P450 oxygenases (29). Therefore, the modulation of P450 1A1 activity has been considered as a potential target for cancer chemoprevention.

The present study is defined as a 'pilot study' since it was performed with 25 clinical cases. The association of AHR expression with tumor grade, although it was strong, needs to be confirmed in a further full-scale experiment. In conclusion, we demonstrated that: i) the majority of hOSCC tissues expressed elevated levels of AHR, CYP1A1 and HSP-90; ii) the intensity and the percentage of positive neoplastic cells increased proceeding from low grade to high grade hOSCCs; iii) in vitro cytotoxic polydatin concentration induced a decrease in AHR, CYP1A1 and HSP-90 protein expression. Finally, molecular simulation studies provided a new rationale use of polydatin and encourage us to continue in vitro studies to support the use of polydatin in oral cancer prevention and/or as alimentary support associated with antitumoral therapy.

\section{Competing interests}

The authors declare that they have no competing interests.

\section{References}

1. Poland A, Palen D and Glover E: Tumour promotion by TCDD in skin of HRS/J hairless mice. Nature 300: 271-273, 1982.

2. Murray IA, Patterson AD and Perdew GH: Aryl hydrocarbon receptor ligands in cancer: Friend and foe. Nat Rev Cancer 14: 801-814, 2014.

3. Meyer BK, Pray-Grant MG, Vanden Heuvel JP and Perdew GH: Hepatitis B virus $X$-associated protein 2 is a subunit of the unliganded aryl hydrocarbon receptor core complex and exhibits transcriptional enhancer activity. Mol Cell Biol 18: 978-88, 1998.

4. Zhou S, Liu J and Chowbay B: Polymorphism of human cytochrome P450 enzymes and its clinical impact. Drug Metab Rev 41: 89-295, 2009.

5. Stanford EA, Ramirez-Cardenas A, Wang Z, Novikov O, Alamoud K, Koutrakis P, Mizgerd JP, Genco CA, Kukuruzinska M, Monti S, et al: Role for the Aryl hydrocarbon receptor and diverse ligands in oral squamous cell carcinoma migration and tumorigenesis. Mol Cancer Res 14: 696-706, 2016.

6. Quattrochi LC and Tukey RH: Nuclear uptake of the Ah (dioxin) receptor in response to omeprazole: Transcriptional activation of the human CYP1A1 gene. Mol Pharmacol 43: 504-508, 1993.

7. Singh SU, Casper RF, Fritz PC, Sukhu B, Ganss B, Girard B Jr, Savouret JF and Tenenbaum HC: Inhibition of dioxin effects on bone formation in vitro by a newly described aryl hydrocarbon receptor antagonist, resveratrol. J Endocrinol 167: 183-195, 2000.

8. Lee JE and Safe S: Involvement of a post-transcriptional mechanism in the inhibition of CYP1A1 expression by resveratrol in breast cancer cells. Biochem Pharmacol 62: 1113-1124, 2001.

9. He H, Zhao Y, Chen X, Zheng Y, Wu X, Wang R, Li T, Yu Q, Jing J, Ma L, et al: Quantitative determination of trans-polydatin, a natural strong anti-oxidative compound, in rat plasma and cellular environment of a human colon adenocarcinoma cell line for pharmacokinetic studies. J Chromatogr B Analyt Technol Biomed Life Sci 855: 145-151, 2007.

10. Henry-Vitrac C, Desmoulière A, Girard D, Mérillon JM and Krisa S: Transport, deglycosylation, and metabolism of trans-piceid by small intestinal epithelial cells. Eur J Nutr 45: 376-382, 2006.

11. Rotches-Ribalta M, Andres-Lacueva C, Estruch R, Escribano E and Urpi-Sarda M: Pharmacokinetics of resveratrol metabolic profile in healthy humans after moderate consumption of red wine and grape extract tablets. Pharmacol Res 66: 375-382, 2012.

12. Ravagnan GP: Extracts from spermatophyte plants with antitumor activity. Euoropean Patent EP1292319B1. Filed May 29, 2001; issued Aug 15, 2002.

13. Wang C, Luo Y, Lu J, Wang Y and Sheng G: Polydatin induces apoptosis and inhibits growth of acute monocytic leukemia cells. J Biochem Mol Toxicol 30: 200-205, 2016.

14. Cao WJ, Wu K, Wang C and Wan DM: Polydatin-induced cell apoptosis and cell cycle arrest are potentiated by Janus kinase 2 inhibition in leukemia cells. Mol Med Rep 13: 3297-3302, 2016.

15. Zhang Y, Zhuang Z, Meng Q, Jiao Y, Xu J and Fan S: Polydatin inhibits growth of lung cancer cells by inducing apoptosis and causing cell cycle arrest. Oncol Lett 7: 295-301, 2014.

16. De Maria S, Scognamiglio I, Lombardi A, Amodio N, Caraglia $\mathrm{M}$, Cartenì $\mathrm{M}$, Ravagnan $\mathrm{G}$ and Stiuso $\mathrm{P}$ : Polydatin, a natural precursor of resveratrol, induces cell cycle arrest and differentiation of human colorectal Caco-2 cell. J Transl Med 11: 264, 2013.

17. Hogg SJ, Chitcholtan K, Hassan W, Sykes PH and Garrill A: Resveratrol, acetyl-resveratrol, and polydatin exhibit antigrowth activity against $3 \mathrm{D}$ cell aggregates of the SKOV-3 and OVCAR-8 ovarian cancer cell lines. Obstet Gynecol Int 2015: 279591, 2015.

18. Hughes D, Guttenplan JB, Marcus CB, Subbaramaiah K and Dannenberg AJ: Heat shock protein 90 inhibitors suppress aryl hydrocarbon receptor-mediated activation of $C Y P 1 A 1$ and CYP1B1 transcription and DNA adduct formation. Cancer Prev Res 1: 485-493, 2008.

19. Tsuji N, Fukuda N, Nagata Y, Okada H, Haga A, Hatakeyama S, Yoshida S, Okamoto T, Hosaka M, Sekine K, et al: The activation mechanism of the aryl hydrocarbon receptor $(\mathrm{AhR})$ by molecular chaperone HSP-90. FEBS Open Bio 4: 796-803, 2014. 
20. Salzano M, Marabotti A, Milanesi L and Facchiano A: Human aryl-hydrocarbon receptor and its interaction with dioxin and physiological ligands investigated by molecular modelling and docking simulations. Biochem Biophys Res Commun 413: 176-181, 2011.

21. Bryne M, Koppang HS, Lilleng R, Stene T, Bang G and Dabelsteen E: New malignancy grading is a better prognostic indicator than Broder's grading in oral squamous cell carcinomas. J Oral Pathol Med 18: 432-437, 1989.

22. Martano M, Carella F, Squillacioti C, Restucci B, Mazzotta M, Lo Muzio L and Maiolino P: Metallothionein expression in canine cutaneous apocrine gland tumors. Anticancer Res 32: 747-752, 2012.

23. John K, Lahoti TS, Wagner K, Hughes JM and Perdew GH: The $\mathrm{Ah}$ receptor regulates growth factor expression in head and neck squamous cell carcinoma cell lines. Mol Carcinog 53: 765-76, 2014.

24. DiNatale BC, Smith K, John K, Krishnegowda G, Amin SG and Perdew GH: Ah receptor antagonism represses head and neck tumor cell aggressive phenotype. Mol Cancer Res 10: 1369-1379, 2012.

25. Yin XF, Chen J, Mao W, Wang YH and Chen MH: Downregulation of aryl hydrocarbon receptor expression decreases gastric cancer cell growth and invasion. Oncol Rep 30: 364-370, 2013.

26. Tsai CH, Li CH, Cheng YW, Lee CC, Liao PL, Lin CH, Huang SH and Kang JJ: The inhibition of lung cancer cell migration by AhR-regulated autophagy. Sci Rep 7: 41927, 2017.
27. Brooks $J$ and Eltom SE: Malignant transformation of mammary epithelial cells by ectopic overexpression of the aryl hydrocarbon receptor. Curr Cancer Drug Targets 11: 654-669, 2011.

28. Al-Dhfyan A, Alhoshani A and Korashy HM: Aryl hydrocarbon receptor/Cytochrome P450 1A1 pathway mediates breast cancer stem cells expansion through PTEN inhibition and $\beta$-Catenin and Akt activation. Mol Cancer 16: 69, 2017.

29. Androutsopoulos VP, Tsatsakis AM and Spandidos DA: Cytochrome P450 CYP1A1: Wider roles Androutsopoulos in cancer progression and prevention. BMC Cancer 9: 187, 2009.

30. Moyer BJ, Rojas IY, Murray IA, Lee S, Hazlett HF, Perdew GH and Tomlinson CR: Indoleamine 2,3-dioxygenase 1 (IDO1) inhibitors activate the aryl hydrocarbon receptor. Toxicol Appl Pharmacol 323: 74-80, 2017.

31. Hamidullah, Kumar R, Saini KS, Kumar A, Kumar S, Ramakrishna E, Maurya R, Konwar R and Chattopadhyay N: Quercetin-6-C- $\beta$-D-glucopyranoside, natural analog of quercetin exhibits anti-prostate cancer activity by inhibiting Akt-mTOR pathway via aryl hydrocarbon receptor. Biochimie 119: 68-79, 2015.

32. Chang CH, Lee CY, Lu CC, Tsai FJ, Hsu YM, Tsao JW, Juan YN, Chiu HY, Yang JS and Wang CC: Resveratrol-induced autophagy and apoptosis in cisplatin-resistant human oral cancer CAR cells: A key role of AMPK and Akt/mTOR signaling. Int J Oncol 50: 873-882, 2017. 